Publ. RIMS Kyoto Univ.

Vol. 6 (1970/71), 477-482

\title{
A Remark on Bures Distance Function for Normal States
}

By

Huzihiro AraKI

\begin{abstract}
An inequality between the norm of the difference of two vector states of a $W *$-algebra and the infimum distance between vectors representing the states in a fixed representation is derived.
\end{abstract}

For a rormal state $\omega$ of a $W^{*}$-algebra $R$ and a normal representation $\pi$ of $R$ on a Hilbert space $H_{\pi}, S(\pi, \omega)$ denotes the set of all vectors $x$ in $H_{\pi}$ such trat $(x, \pi(Q) x)=\omega(Q)$ for all $Q \in R$. (We do not assume $\omega(\mathbb{1})=\mathbb{1}$ in accordance with Bures.) Let

$$
\mathrm{d}_{\pi}\left(\omega, \omega^{\prime}\right)=\inf \left\{\|x-y\| ; x \in \mathrm{S}(\pi, \omega), y \in \mathrm{S}\left(\pi, \omega^{\prime}\right)\right\}
$$

whenever $\mathrm{S}(\pi, \omega)$ and $\mathrm{S}\left(\pi, \omega^{\prime}\right)$ are non-empty and $\mathrm{d}_{\pi}\left(\omega, \omega^{\prime}\right)=\sqrt{2}$ otherwise. Leî

$$
\mathrm{d}\left(\omega, \omega^{\prime}\right)=\inf _{\pi} \mathrm{d}_{\pi}\left(\omega, \omega^{\prime}\right) .
$$

A trivial calculation shows

$$
\mathrm{d}\left(\omega, \omega^{\prime}\right) \geqq\left[\omega(1)+\omega^{\prime}(1)\right]^{-1}\left\|\omega-\omega^{\prime}\right\| .
$$

Bures has shown [1] that

$$
\mathrm{d}\left(\omega, \omega^{\prime}\right)^{2} \leqq\left\|\omega-\omega^{\prime}\right\| .
$$

We shall derive a similar inequality for a fixed representation $\pi$, which implies the equivalence of topologies induced by the norm and $\mathrm{d}_{\pi}\left(\omega, \omega^{\prime}\right)$ on the set of all vector states in the representation $\pi$.

If $R$ is a von Neumann algebra on a Hilbert space $H$ and $x \in H$, ve write $\omega_{\imath}(Q)=(x, Q x)$ for $Q \in R$. If $\pi$ is a representation of $R$ on $H_{\pi}$ and $x \in H_{\pi}$, we also write $\omega_{2}(Q)=(x, \pi(Q) x)$ for $Q \in R$.

Received September 30, 1970. 
Our aim is the proof of the following:

Theorem 1. For all $x, y \in H_{\pi}$,

$$
\mathrm{d}_{\pi}\left(\omega_{x}, \omega_{y}\right)^{2} \leqq 2\left\|\omega_{x}-\omega_{y}\right\|
$$

We start with technical lemmas.

Lemma 2. $\omega_{x}=\omega_{y}$ if and only if there exists a partial isometry $W \in R^{\prime}$ such that $W x=y, W^{*} y=x, W H=\overline{R y}, W^{*} H=\overline{R x}$.

This is essentially Lemma 3, Chap. I, $\$ 4.1$ in [2] where $\Re=\overline{R x}$, $\Re_{1}=\overline{R y}, \mathscr{B}=R\left|\Re, \mathscr{B}_{1}=R\right| \Re_{1}, \bar{\Phi}$ and $\Phi_{1}$ are restriction maps of $R$ to $\Omega$ and $\Omega_{1}$.

Lemma 3. Let $\left\{F_{\alpha}\right\}$ be a partition of 1 by central projections of $\pi(R)$. For $x, y \in H_{\pi}$,

$$
\begin{aligned}
& \left\|\omega_{x}-\omega_{y}\right\|=\sum_{\alpha}\left\|\omega_{F_{\alpha} x}-\omega_{F_{\alpha} y}\right\|, \\
& \mathrm{d}_{\pi}\left(\omega_{x}, \omega_{y}\right)^{2}=\sum_{\alpha} \mathrm{d}_{\pi}\left(\omega_{F_{\alpha} x}, \omega_{F_{\alpha} y}\right)^{2} .
\end{aligned}
$$

Proof. For the norm, the following computation proves (2)

$$
\begin{aligned}
\left\|\omega_{x}-\omega_{y}\right\| & =\sup \left\{\operatorname{Re}\left[\omega_{x}(Q)-\omega_{y}(Q)\right] ;\|Q\| \leqq 1, Q \in \pi(R)\right\} \\
& =\sup \left\{\operatorname{Re} \sum_{\alpha}\left[\omega_{F_{\alpha} x}\left(F_{\alpha} Q\right)-\omega_{F_{\alpha} y}\left(F_{\alpha} Q\right)\right] ;\|Q\| \leqq 1, Q \in \pi(R)\right\} \\
& =\sum_{\alpha} \sup \left\{\operatorname{Re}\left[\omega_{F_{\alpha} x}\left(Q_{\alpha}\right)-\omega_{F_{\alpha} y}\left(Q_{\alpha}\right)\right] ;\left\|Q_{\alpha}\right\| \leqq 1, Q_{\alpha} \in \pi(R) F_{\alpha}\right\} \\
& =\sum_{\alpha} \sup \left\{\operatorname{Re}\left[\omega_{F_{\alpha} x}(Q)-\omega_{F_{\alpha} y}(Q)\right] ;\|Q\| \leqq 1, Q \in \pi(R)\right\} \\
& =\sum_{\alpha}\left\|\omega_{F_{\alpha} x}-\omega_{F_{\alpha} y}\right\| .
\end{aligned}
$$

For $\mathrm{d}_{\pi}$, we denote the representation $F_{\alpha}(Q)$ of $Q \in R$ on $F_{\alpha} H_{\pi}$ by $F_{\alpha} \pi$. Then,

$$
\begin{aligned}
\mathrm{d}_{\pi}\left(\omega_{x}, \omega_{y}\right)^{2} & =\inf \left\{\left\|x^{\prime}-y^{\prime}\right\|^{2} ; x^{\prime} \in \mathrm{S}\left(\pi, \omega_{x}\right), y^{\prime} \in \mathrm{S}\left(\pi, \omega_{y}\right)\right\} \\
& =\inf \left\{\sum_{\alpha}\left\|F_{\alpha} x^{\prime}-F_{\alpha} y^{\prime}\right\|^{2} ; x^{\prime} \in \mathrm{S}\left(\pi, \omega_{x}\right), y^{\prime} \in \mathrm{S}\left(\pi, \omega_{y}\right)\right\} \\
& =\sum_{\alpha} \inf \left\{\left\|x_{\alpha}^{\prime}-y_{\alpha}^{\prime}\right\|^{2} ; x_{\alpha}^{\prime} \in \mathrm{S}\left(F_{\alpha} \pi, \omega_{F_{\alpha} x}\right), y_{\alpha}^{\prime} \in \mathrm{S}\left(F_{\alpha} \pi, \omega_{F_{\alpha} y}\right)\right\} \\
& =\sum_{\alpha} \inf \left\{\left\|x_{\alpha}^{\prime}-y_{\alpha}^{\prime}\right\|^{2} ; x_{\alpha}^{\prime} \in \mathrm{S}\left(\pi, \omega_{F_{\alpha} x}\right), y_{\alpha}^{\prime} \in \mathrm{S}\left(\pi, \omega_{F_{\alpha} y}\right)\right\} \\
& =\sum_{\alpha} \mathrm{d}_{\pi}\left(\omega_{F_{\alpha} x}, \omega_{F_{\alpha} y}\right)^{2} .
\end{aligned}
$$

Corollary 4. Let $x^{\prime} \in \mathrm{S}\left(\pi, \omega_{x}\right), y^{\prime} \in \mathrm{S}\left(\pi, \omega_{y}\right), \varepsilon>0$ and 


$$
\left\|x^{\prime}-y^{\prime}\right\|^{2}<\mathrm{d}_{\pi}\left(\omega_{x}, \omega_{y}\right)^{2}+\varepsilon .
$$

Let $F$ be a central projection of $\pi(R)$. Then

$$
\left\|F x^{\prime}-F y^{\prime}\right\|^{2}<\mathrm{d}_{\pi}\left(\omega_{F x}, \omega_{F y}\right)^{2}+\varepsilon .
$$

Proof. From (4) and Lemma 3, we have

$$
\begin{aligned}
& \left\|F x^{\prime}-F y^{\prime}\right\|^{2}+\left\|(1-F) x^{\prime}-(1-F) y^{\prime}\right\|^{2} \\
& \quad<\mathrm{d}_{\pi}\left(\omega_{F x}, \omega_{F y}\right)^{2}+\mathrm{d}_{\pi}\left(\omega_{(1-F) x}, \omega_{(1-F) y}\right)^{2}+\varepsilon .
\end{aligned}
$$

On the other hand, we have $F x^{\prime} \in \mathrm{S}\left(\pi, \omega_{F x}\right), F y^{\prime} \in \mathrm{S}\left(\pi, \omega_{F y}\right)$, and similar equations for $1-F$. Therefore,

$$
\begin{aligned}
& \left\|F x^{\prime}-F y^{\prime}\right\|^{2} \geqq \mathrm{~d}_{\pi}\left(\omega_{F x}, \omega_{F y}\right)^{2}, \\
& \left\|(1-F) x^{\prime}-(1-F) y^{\prime}\right\|^{2} \geqq \mathrm{~d}_{\pi}\left(\omega_{(1-F) x}, \omega_{(1-F) y}\right)^{2} .
\end{aligned}
$$

Hence we have (5).

Q.E.D.

Lemma 5. For $x, y, z \in H_{\pi}$,

$$
\mathrm{d}_{\pi}\left(\omega_{x}, \omega_{y}\right)+\mathrm{d}_{\pi}\left(\omega_{y}, \omega_{z}\right) \geqq \mathrm{d}_{\pi}\left(\omega_{x}, \omega_{z}\right) .
$$

Proof. Let $\varepsilon>0$. Let $\alpha_{\beta} \in H_{\pi}, \alpha, \beta=x, y, z$ be such that $\alpha_{\beta}$ $\in \mathrm{S}\left(\pi, \omega_{\alpha}\right)$ and

$$
\left\|\alpha_{\beta}-\beta_{\alpha}\right\|^{2}<\mathrm{d}_{\pi}\left(\omega_{\alpha}, \omega_{\beta}\right)^{2}+\varepsilon .
$$

By Lemma 2, there exists a partial isometry $W$ in $R^{\prime}$ such that $W y_{x}=y_{z}, W^{*} y_{z}=y_{x}$. There exists then a central projection $F$ of $R^{\prime}$ and partial isometries $W_{1}$ and $W_{2}$ in $R^{\prime}$ such that

$$
\begin{aligned}
& F\left(1-W W^{*}\right)=W_{1}^{*} W_{1}, \quad F\left(1-W^{*} W\right) \geqq W_{1} W_{1}^{*}, \\
& (1-F)\left(1-W^{*} W\right)=W_{2}^{*} W_{2}, \quad(1-F)\left(1-W W^{*}\right) \geqq W_{2} W_{2}^{*} .
\end{aligned}
$$

Let

$$
W_{1}^{\prime}=\left(W_{1}+W^{*}\right) F, \quad W_{2}^{\prime}=\left(W_{2}+W\right)(1-F) .
$$

Then $W_{1}^{\prime}$ and $W_{2}^{\prime}$ are isometric on $F H$ and $(1-F) H$ and satisfies

$$
W_{1}^{\prime} y_{z}=F y_{x}, \quad W_{2}^{\prime} y_{x}=(1-F) y_{z} .
$$

Let

$$
x^{\prime}=F x_{y}+W_{2}^{\prime} x_{y}, y^{\prime}=F y_{x}+(1-F) y_{z}, z^{\prime}=W_{1}^{\prime} z_{y}+(1-F) z_{y} .
$$

Then we obtain $x^{\prime} \in \mathrm{S}\left(\pi, \omega_{x}\right), y^{\prime} \in \mathrm{S}\left(\pi, \omega_{y}\right), z^{\prime} \in \mathrm{S}\left(\pi, \omega_{z}\right)$ and 


$$
\begin{aligned}
& \left\|x^{\prime}-y^{\prime}\right\|^{2}=\left\|F\left(x_{y}-y_{x}\right)\right\|^{2}+\left\|(1-F)\left(x_{y}-y_{x}\right)\right\|^{2}=\left\|x_{y}-y_{x}\right\|^{2}, \\
& \left\|y^{\prime}-z^{\prime}\right\|^{2}=\left\|F\left(y_{z}-z_{y}\right)\right\|^{2}+\left\|(1-F)\left(y_{z}-z_{y}\right)\right\|^{2}=\left\|y_{z}-z_{y}\right\|^{2},
\end{aligned}
$$

where (8) and isometry of $W_{1}^{\prime}$ and $W_{2}^{\prime}$ on $F H$ and $(1-\mathbb{F}) H$ are used.

From (9), (10) and (7), we obtain

$$
\begin{aligned}
\mathrm{d}_{\pi}\left(\omega_{x}, \omega_{z}\right) & \leqq\left\|x^{\prime}-z^{\prime}\right\| \leqq\left\|x^{\prime}-y^{\prime}\right\|+\left\|y^{\prime}-z^{\prime}\right\| \\
& \leqq \mathrm{d}_{\pi}\left(\omega_{x}, \omega_{y}\right)+\mathrm{d}_{\pi}\left(\omega_{y}, \omega_{z}\right)+\varepsilon^{\prime}(\varepsilon)
\end{aligned}
$$

where $\varepsilon^{\prime}(\varepsilon) \rightarrow 0$ as $\varepsilon \rightarrow 0$. Hence we have (6).

Q.E.D.

Lemma 6. For $x \in H_{\pi}$ and $Q \in \pi(R)^{+}$,

$$
\mathrm{d}_{\pi}\left(\omega_{x}, \omega_{Q \tau}\right)^{2} \leqq\left\|\omega_{x}-\omega_{Q x}\right\| \text {. }
$$

If $\omega_{x} \leqq n \omega_{y}$ for some $n>0$, then (11) holds.

Proof. Due to the proof of Proposition 1.12 of [1].

Lemma 7. For $x \in H_{\pi}$ and $Q \in \pi\left(R^{\prime}\right)$, (11) holds.

Proof. For $Q_{1} \in \pi(R)$, we have

$$
\omega_{Q_{x}}\left(Q_{1}^{*} Q_{1}\right)=\left\|Q Q_{1} x\right\|^{2} \leqq\|Q\|^{2} \omega_{x}\left(Q_{1}^{*} Q_{1}\right) .
$$

Hence Lemma 6 implies (11).

Q.E.D.

Proof of Theorem 1. Let $e_{1}$ and $e_{2}$ be an orthonormal basis of $M$. Let $\pi^{\prime}$ be the representation $\pi^{\prime}(Q)=\pi(Q) \otimes 1$ on $H^{\prime}=H_{\pi} \otimes M$. Let $0<\varepsilon<1$ and

$$
z(\varepsilon)=(1-\varepsilon)^{1 / 2} x \otimes e_{1}+\varepsilon^{1 / 2} y \otimes e_{2} .
$$

Since $\varepsilon^{-1} \omega_{z(\varepsilon)} \geqq \omega_{y \smile e_{2}}$, there exists $A \in \pi(R)^{+}$such that $\hat{A}=A \otimes 1$ satisfies $\omega_{\widehat{A}(\varepsilon)}=\omega_{y}$, by Sakai's Radon Nikodym theorem [3]. By Lemma 2, there exists a partial isometry $W$ in $\pi^{\prime}(R)^{\prime}$ such that $W\left(y \otimes e_{1}\right)=\widehat{A} z(\varepsilon)$. By the proof of Proposition 1.12 in [1], we have

$$
\|\widehat{A} z(\varepsilon)-z(\varepsilon)\|^{2} \leqq\left\|\omega_{y}-\omega_{z(\varepsilon)}\right\| .
$$

Let $U_{i j}$ be defined by $\left(\Phi, U_{i j} \Psi\right)=\left(\Phi \otimes e_{i}, W\left(\Psi \otimes e_{j}\right)\right)$ for all $\Phi, \Psi \in H_{\pi}$. Then $U_{i j} \in \pi(R)^{\prime}$ due to $W \in \pi^{\prime}(R)^{\prime}=(\pi(R) \otimes 1)^{\prime}$. Further, $\left(W^{*} W\right)$ $\cdot\left(y \otimes e_{1}\right)=y \otimes e_{1}$ implies $\left(U_{11}^{*} U_{11}+U_{21}^{*} U_{21}\right) y=y$. Let $y^{\prime}=U_{11} y, y^{\prime \prime}=U_{21} y$. We then have 


$$
\omega_{y}=\omega_{y^{\prime}}+\omega_{y \prime \prime}
$$

The equation (12) now reads as

$$
\begin{aligned}
\left\|\omega_{y}-\omega_{z(\varepsilon)}\right\| & \geqq\left\{\left\|W\left(y \otimes e_{1}\right)-x \otimes e_{1}\right\|-\left\|z(\varepsilon)-x \otimes e_{1}\right\|\right\}^{2} \\
& \geqq\left\|y^{\prime}-x\right\|^{2}+\left\|y^{\prime \prime}\right\|^{2}-\varepsilon^{\prime}(\varepsilon)
\end{aligned}
$$

where $\varepsilon^{\prime}(\varepsilon) \rightarrow 0$ as $\varepsilon \rightarrow 0$. We also have

$$
\left\|\omega_{y}-\omega_{\lambda_{1}(\varepsilon)}\right\| \leqq\left\|\omega_{y}-\omega_{2}\right\|+\left\|\omega_{x}-\omega_{z(\varepsilon)}\right\| \leqq\left\|\omega_{y}-\omega_{x}\right\|+\varepsilon^{\prime \prime}(\varepsilon)
$$

where $\varepsilon^{\prime \prime}(\varepsilon) \rightarrow 0$ as $\varepsilon \rightarrow 0$.

By Lemma 6, we have from (13),

$$
\mathrm{d}_{\pi}\left(\omega_{y}, \omega_{y^{\prime}}\right)^{2} \leqq\left\|\omega_{y}-\omega_{y^{\prime}}\right\|=\left\|\omega_{y^{\prime \prime}}\right\|=\left\|y^{\prime \prime}\right\|^{2} .
$$

By definition,

$$
\mathrm{d}_{\pi}\left(\omega_{y^{\prime}}, \omega_{x}\right)^{2} \leqq\left\|y^{\prime}-x\right\|^{2}
$$

By Lemma 5, we have

$$
\begin{aligned}
\mathrm{d}_{\pi}\left(\omega_{x}, \omega_{y}\right)^{2} & \leqq\left\{\mathrm{~d}_{\pi}\left(\omega_{x}, \omega_{y^{\prime}}\right)+\mathrm{d}_{\pi}\left(\omega_{y^{\prime}}, \omega_{y}\right)\right\}^{2} \\
& \leqq 2 \mathrm{~d}_{\pi}\left(\omega_{x}, \omega_{y^{\prime}}\right)^{2}+2 \mathrm{~d}_{\pi}\left(\omega_{y^{\prime}}, \omega_{y}\right)^{2} .
\end{aligned}
$$

Collecting $(14) \sim(18)$ together, and taking the limit of $s \rightarrow 0$, we have (1).

Q.E.D.

Remark. If $\pi(R)^{\prime}$ is properly infinite, then $\omega_{z(\varepsilon)}$ in the above proof can be realized as a vector state in $H_{\pi}$. Hence we immediately obtain an improved version of the inequality:

$$
\mathrm{d}_{\pi}\left(\omega_{x}, \omega_{y}\right)^{2} \leqq\left\|\omega_{x}-\omega_{y}\right\|
$$

If $\pi(R)$ is finite and has a trace vector $\varphi$ in $H_{\pi}$, then (19) can be proved as follows:

By the proof of Theorem 1 and Lemma $5, \mathrm{~d}_{\pi}\left(\omega_{x}, \omega_{y}\right)$ is continuous in $\omega_{x}$ and $\omega_{y}$ relative to the norm topology on states. Hence it is enough to prove (19) for a dense set of states $\omega_{x}$ and $\omega_{y}$. We shall consider vector states $\omega_{A \rho}$ and $\omega_{B \rho}, A, B \in \pi(R)^{+}$, which are dense in the set of all normal states of $\pi(R)$ and hence in the set of $\pi$-vector states of $R$.

Let $E_{+}$and $E_{-}$be spectral projection of $A-B$ for $(0, \infty)$ and 
$(-\infty, 0)$. For $Q=E_{+}-E_{-}$, we have $\|Q\|=1, Q(A-B)=(A-B) Q$ $=|A-B|$. Since $\omega_{\varphi}$ is a trace, we have

$$
\begin{aligned}
\omega_{A \varphi}(Q) & -\omega_{B \varphi}(Q)=\omega_{\varphi}\left(Q\left(A^{2}-B^{2}\right)\right) \\
& =\frac{1}{2}\left\{\omega_{\varphi}(Q(A-B)(A+B))+\omega_{\varphi}((A-B) Q(A+B))\right\} \\
& =\omega_{\varphi}(|A-B|(A+B)) \\
& =\omega_{|A-B|^{1 / 2}}\left(E_{+}(A+B) E_{+}+E_{-}(A+B) E_{-}\right) .
\end{aligned}
$$

Since $E_{+}(A+B) E_{+} \geqq E_{+}(A-B) E_{+}=E_{+}|A-B| \quad$ and $\quad E_{-}(A+B) E_{-}$ $\geqq E_{-}(B-A) E_{-}=|A-B| E_{-}$, we obtain

$$
\begin{aligned}
\left\|\omega_{A \varphi}-\omega_{B \varphi}\right\| & \geqq \omega_{\varphi}\left(|A-B|^{2}\right)=\|A \varphi-B \varphi\|^{2} \\
& \geqq \mathrm{~d}_{\pi}\left(\omega_{A \varphi}-\omega_{B \varphi}\right) .
\end{aligned}
$$

Combining above conclusions, we see that if the finite part $F_{\pi}(R)$ of $\pi(R)$ is not smaller than its commutant $F_{\pi}(R)^{\prime}$, then (19) holds.

On the other hand, (19) is not true in general as can be seen from an example of $\pi(R)=\mathscr{B}\left(H_{\pi}\right), \operatorname{dim} H_{\pi}=2$.

\section{References}

[1] Bures, D. J. C., Trans. Amer. Math. Soc. 135 (1969), 199-212.

[2] Dixmier, J., Les algèbres d'opérateurs dans l'espace hilbertien. 2nd ed., GauthierVillars, Paris, 1969.

[3] Sakai, S., Bull. Amer. Math. Soc. 71 (1965), 149-151. 